\title{
Ursodeoxycholic acid in chronic liver disease
}

\author{
J S de Caestecker, R P Jazrawi, M L Petroni, T C Northfield
}

\begin{abstract}
The hydrophilic bile acid ursodeoxycholic acid has recently been shown to reduce biochemical markers of both cholestasis and hepatocellular damage in patients with chronic liver diseases. The most compelling evidence available is for chronic cholestatic liver diseases, in particular primary biliary cirrhosis, primary sclerosing cholangitis, and cholestasis associated with cystic fibrosis. The effects may be less beneficial in patients with advanced liver disease from these conditions. Data from placebo controlled trials are now available in support of earlier uncontrolled observations, but it is not yet clear whether short term benefit results in an improvement in longterm prognosis. The mechanism of action of the compound seems to reside in its displacement of toxic hydrophobic bile acids from both the bile acid pool and hepatocellular membranes. There may be an independent effect on bile flow, which could be of particular importance in cystic fibrosis, and possibly an effect on the immune system. Ursodeoxycholic acid should now be regarded as occupying a central place in the medical management of chronic cholestatic liver diseases, in particular primary biliary cirrhosis, because it improves cholestasis and reduces hepatocellular damage and it is not toxic. Research should now be targeted on whether treatment with ursodeoxycholic acid, initiated early in cholestatic liver conditions, improves the longterm outcome.
\end{abstract}

The bile acid ursodeoxycholic acid occurs naturally in small quantities in human bile $(<4 \%$ of total bile acids). It is formed by $7-\beta$ epimerisation of the primary bile acid chenodeoxycholic acid through the action of intestinal bacteria. It was first isolated earlier this century from the bile of the Chinese black bear, after which species it was named. ' Nearly 40 years ago it was manufactured synthetically in Japan, finding use as a treatment for a wide range of liver diseases. Its popularity stemmed not from rigorous scientific study but from a longstanding tradition of the beneficial properties of black bear bile ('Yutan').' More general interest in the compound was aroused 15 years ago when Japanese investigators reported that ursodeoxycholic acid desaturated bile and dissolved cholesterol gall stones. ${ }^{12}$

Leuschner and colleagues were the first to report in an English language journal improvement in liver function tests in patients with liver disease. ${ }^{3}$ As a chance finding, they observed this improvement in a group of patients with chronic liver disease who were receiving ursodeoxycholic acid to dissolve gall stones. This report stimulated interest in the compound as a treatment for a variety of adult and childhood liver disorders, principally those resulting in cholestasis. The result has been a large number of uncontrolled studies, with the preliminary results of controlled studies now beginning to emerge. This review attempts to place in perspective the role of ursodeoxycholic acid in the treatment of liver disease, with discussion of recent data on its mechanism of action. The aim will be to conclude what implications arise for current clinical practice and future research.

\section{Clinical studies}

\section{PRIMARY BILIARY CIRRHOSIS}

Primary biliary cirrhosis is an example of a disease in which two different processes may be injuring the liver. ${ }^{4}$ One is the immunological injury to intrahepatic bile ducts by mechanisms which are still poorly understood. ${ }^{5}$ Most drugs that have been evaluated for treatment of primary biliary cirrhosis have been used in an attempt either to influence this abnormal immunological response or to inhibit the fibrosis consequent on chronic inflammatory damage. Although some, such as colchicine, cyclosporin, and methotrexate seem promising and are still being evaluated, use of others such as penicillamine and prednisolone has now been abandoned..$^{56}$

The second mechanism, possibly a consequence of the first, is the retention of hydrophobic endogenous bile acids, including chenodeoxycholic, deoxycholic, and lithocholic acids (see Figure). These are damaging to cellular membranes, such as the bile canalicular membrane $^{7}$ and may thus aggravate the cholestasis. ${ }^{14}$ Fisher and Paradine were the first to report a beneficial effect of ursodeoxycholic acid in patients with primary biliary cirrhosis, ${ }^{8}$ followed by Poupon and colleagues in a report published in the Lancet and attracting much interest. ${ }^{9}$ The latter group observed in an open study improvement in both symptoms and serum enzyme markers of cholestasis with ursodeoxycholic acid given over a two year treatment period, with subsequent deterioration when it was discontinued. Several uncontrolled studies have confirmed these original observations, ${ }^{10-14}$ but all have been small studies with fewer than 20 patients. Nevertheless, there has been a striking uniformity in these reports of improvement in symptoms and serum transaminases as well as alkaline phosphatase and $\gamma$ glutamyltransferase. All enzyme levels were reported decreased to $30-70 \%$ of pretreatment values. Glutamine dehydrogenase, a hepatocyte mitochondrial enzyme and a specific indicator of hepatocyte damage, is also decreased. ${ }^{15}$ Thus, serum enzyme markers of both cholestasis and hepatocellular damage are reduced on treatment. 


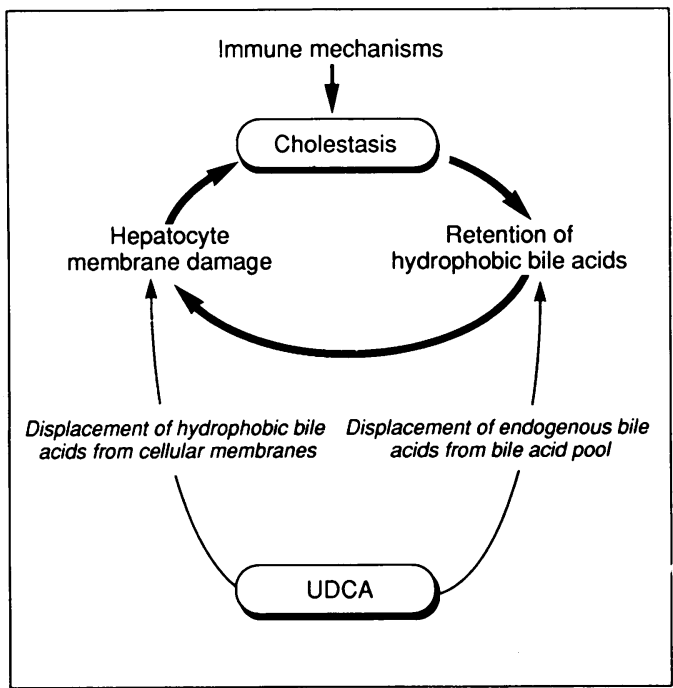

Putative pathophysiology of primary biliary cirrhosis and mechanisms of action of ursodeoxycholic acid (UDCA). Bold arrows indicate an increased effect, light arrows a decreased effect.

These encouraging results are now being confirmed in controlled studies, ${ }^{15-21}$ including three large multicentre studies. ${ }^{172} 21$ The results from four trials ${ }^{15161821}$ giving sufficient information are summarised in the Table. These include the two which have appeared as full papers and two abstracts. Only in the two full papers ${ }^{1521}$ is sufficient information supplied to determine that the active and placebo groups are well matched (Table). Some of the reports are based on interim analysis at an interval of six months ${ }^{172021}$ and others are only short term studies, though one has given results after two years of treatment. ${ }^{16}$ Although in some of the open studies symptoms were reported to improve during ursodeoxycholic acid treatment, several of the controlled studies have found that symptoms such as pruritus and fatigue, though reduced, are diminished to the same extent with ursodeoxycholic acid as with placebo..$^{1521}$ The maximum reduction in serum biochemical parameters occurs during the first year of treatment. ${ }^{16}$ Hadziyannis and colleagues found that, particularly in patients with stages III and IV of the disease, biochemical and symptomatic deterioration occurred during the second year of treatment. ${ }^{16}$ This complements other uncontrolled observations that patients with late stage disease either respond poorly or may even deteriorate on ursodeoxycholic acid. ${ }^{22}{ }^{23}$ Serum IgM decreases during treatment, ${ }^{15}$ 19-21 hinting at an effect of ursodeoxycholic acid on the immune process, although no changes in antimitochondrial antibody titre have been observed in these reports. Finally, histological improvement in liver biopsy specimens has been reported in four studies, notably a reduction in inflammation, bile ductular proliferation, and epithelial cell proliferation (Table). ${ }^{15181921}$

\section{OTHER CHOLESTATIC LIVER DISEASES}

Ursodeoxycholic acid treatment has been tried in a variety of other liver conditions. Symptoms and serum enzyme markers of cholestasis and hepatocellular damage in primary sclerosing cholangitis have been shown to improve in both open $^{2+25}$ and controlled studies. ${ }^{26}$ Inevitably, numbers have been small in these studies because the disease is rare. In the cholestatic liver disease associated with cystic fibrosis, average reductions in alanine transaminase of $60 \%$ and alkaline phosphatase of $47 \%$ were observed after six months of treatment with ursodeoxycholic acid in an open study. ${ }^{27}$ This study also reported an improvement in nutritional state on treatment despite a lack of effect on steatorrhoea. The improvement in transaminases in this condition has been confirmed in a placebo controlled trial. ${ }^{28}$ In patients undergoing surgery for obstructive jaundice, a randomised trial of ursodeoxycholic acid administration before surgery did not show any benefit in terms of endotoxaemia, renal failure, or postoperative morbidity and mortality. ${ }^{29} \mathrm{~A}$ short term (three months) controlled trial in children suffering from a variety of cholestatic liver diseases was reported to benefit neither symptoms nor biochemical parameters. ${ }^{30}$

There are reports suggesting that ursodeoxycholic acid may be administered with benefit to children who have had biliary reconstructions

Summary of some controlled studies of ursodeoxycholic acid in primary biliary cirrhosis

\begin{tabular}{|c|c|c|c|c|c|c|c|c|c|c|}
\hline \multirow[b]{2}{*}{ Study } & \multirow[b]{2}{*}{$\begin{array}{l}\text { No of } \\
\text { subjects }\end{array}$} & \multirow[b]{2}{*}{$\begin{array}{l}\text { Ursodeoxycholic } \\
\text { acid dose }(\mathrm{mg} / \mathrm{kg} / \\
\text { day) }\end{array}$} & \multirow{2}{*}{$\begin{array}{l}\text { Histological } \\
\text { stage } \\
\text { (No of } \\
\text { subjects) }\end{array}$} & \multirow[b]{2}{*}{$\begin{array}{l}\text { Time of } \\
\text { analysis } \\
\text { (months) }\end{array}$} & \multicolumn{5}{|c|}{$\%$ change from baseine $\$$} & \multirow[b]{2}{*}{ Histology } \\
\hline & & & & & $\begin{array}{l}\text { Alkaline } \\
\text { phosphatase }\end{array}$ & $\begin{array}{l}\text { Alanine } \\
\text { transaminase }\end{array}$ & $\begin{array}{l}\text { Y glutamyl- } \\
\text { transferase }\end{array}$ & Bilirubin & $\begin{array}{l}\text { Serum } \\
\text { IgM }\end{array}$ & \\
\hline Leuschner et al $(1989)^{15} \dagger$ & 20 & 10 & $\begin{array}{l}\text { I }(7) \ddagger \\
\text { II }(10) \\
\text { III }(3)\end{array}$ & 3 & -55 & -61 & -73 & - & $-30 \%$ & $\begin{array}{l}\text { Two deteriorated, six improved } \\
\text { of } 10 \text { on ursodeoxycholic acid; } \\
\text { four deteriorated, one } \\
\text { improved of } 10 \text { on placebo }\end{array}$ \\
\hline Hadziyannis et al $(1989)^{16}$ & 50 & $10-15$ & $\begin{array}{l}\text { All stages } \\
\text { (no details) }\end{array}$ & $\begin{array}{l}6 \\
12 \\
18-24\end{array}$ & $\begin{array}{l}-42 \\
-42 \\
-37\end{array}$ & $\begin{array}{r}-37 \\
-41 \\
-9\end{array}$ & $\begin{array}{l}-39 \\
-50 \\
-39\end{array}$ & $\begin{array}{r}-34 \\
-26 \\
+7\end{array}$ & - & $\begin{array}{l}\text { Histology on seven patients only } \\
\text { at two years; no change or } \\
\text { deterioration }\end{array}$ \\
\hline O'Brien et al $(1990)^{18}$ & 16 & $8-12$ & $\begin{array}{l}\text { Not } \\
\text { specified }\end{array}$ & 6 & -42 & -47 & -56 & -32 & - & $\begin{array}{l}\text { Appreciable improvement } \\
\text { placebo and baseline in } \\
\text { histological score and grade }\end{array}$ \\
\hline Poupon et al $(1990)^{21}$ & 138 & $13-15$ & $\begin{array}{l}\text { I }(32) \ddagger \\
\text { II }(47) \\
\text { III }(39) \\
\text { IV }(20)\end{array}$ & 6 & -53 & -47 & -61 & -41 & -29 & - \\
\hline
\end{tabular}

${ }^{\star}$ Half received placebo and half received ursodeoxycholic acid. $†$ Trial continued for nine months, although percentage fall in biochemical parameters given at three months. $\ddagger$ Leuschner's and Poupon's studies are the only full papers and only in these is there sufficient data on patient characteristics; in both, placebo and treatment groups contained comparable proportions of histological grades. In Poupon's study, sex, age, and time since diagnosis were similar in both groups. Serum biochemical parameters were not significantly different at entry between placebo and treatment groups in either study, apart from higher alkaline phosphatase ( $\mathrm{p}<0.002$ ) in the treatment group in Poupon's study.

in Pignificant differences in all parameters in ursodeoxycholic acid group $v$ placebo in all studies; however, in Hadziyannis's study no significant changes were noted in patients with late stage primary biliary cirrhosis or in those with serum bilirubin $>171 \mu \mathrm{mol} / \mathrm{l}$; fall in bilirubin not significantly different from placebo in $\mathrm{O}$ 'Brien's study. 
for biliary atresia. ${ }^{31-34}$ These patients suffer from recurrent cholangitis, and it has been found that ursodeoxycholic acid may in some cases improve bile flow, ${ }^{33}$ reduce episodes of cholangitis, ${ }^{3+}$ and result in improved weight gain. ${ }^{31}{ }^{34}$ Anecdotal reports suggest a benefit of ursodeoxycholic acid in cholestasis of pregnancy ${ }^{35}$ and benign recurrent intrahepatic cholestasis. ${ }^{36}$

\section{CHRONIC HEPATITIS}

Ursodeoxycholic acid has also been observed to reduce transaminase levels in patients with chronic hepatitis. ${ }^{31+}$ Two double blind controlled trials have been reported: one, comprising 59 blood donors with raised alanine transaminase, found a significant reduction in alanine transaminase with ursodeoxycholic acid, with reversion to baseline levels when treatment was stopped. ${ }^{37}$ The other study found no benefit of serum enzymes or liver histology in patients with non-A non-B chronic hepatitis ( $85 \%$ had antibodies to hepatitis $\mathrm{C}$ virus) followed up for one year. ${ }^{38}$ This was a small trial (20 patients), however, and large spontaneous fluctuations in alanine transaminase are characteristic of such patients.

Mechanisms of action of ursodeoxycholic acid It is clear from the results of clinical studies that ursodeoxycholic acid results in improvement of enzyme markers reflecting hepatocellular necrosis as well as cholestasis. It is more hydrophilic than the endogenous bile acids chenodeoxycholic acid and deoxycholic acid, though whether it is more hydrophilic than cholic acid is debatable. ${ }^{2}$ It is less surface active than the more hydrophobic bile acids ${ }^{2}$ and thus has less tendency to partition into membranes and to solubilise membrane lipids. ${ }^{39+0}$ Consequently, it is less toxic to a variety of cells studied in vitro, including mast cells, ${ }^{39}$ red blood cells, ${ }^{41}$ and isolated hepatocytes. ${ }^{42+3}$ The most hydrophobic bile acids, as expected, are the most toxic. Furthermore, ursodeoxycholic acid protects against the cytotoxic effects of other hydrophobic bile acids both in vitro and in vivo ${ }^{41-43}$ by stabilising the cell membrane into which bile acids seem to be incorporated. ${ }^{15}$

The effects of bile acids in intact animals have been elegantly shown in a series of experiments from Kitani's group, ${ }^{+16}$ who found that infusions of hydrophobic salts in increasing concentrations produced an initial choleresis followed by profound cholestasis indicated by a fall in bile flow and bile acid secretion rate. Coadministration of ursodeoxycholic acid, however, inhibited cholestasis even when a hydrophobic bile acid was infused at high rates. The same group observed that ursodeoxycholic acid prevented biliary leakage of lactate dehydrogenase and albumin induced by hydrophobic bile acids in the rat, indicating that damage to bile duct epithelium was being prevented. ${ }^{+6}$ This protective effect is still seen if the concentration of ursodeoxycholic acid is a quarter that of the hydrophobic bile salt, suggesting it is not simply due to dilution of the more toxic bile sale.' Prevention of cholestasis may simply be a result of the more general membrane protecting effect of ursodeoxycholic acid (discussed above) exerted on the bile canalicular membrane. Indeed, it protects artificial membranes only if they are rich in cholesterol, ${ }^{47}$ as is the case for the bile canalicular membrane.

Erlinger and Dumont have shown that unconjugated ursodeoxycholic acid is capable of producing a bicarbonate rich hypercholeresis, ${ }^{48}$ which could be the result of either cholehepatic recycling of protonated ursodeoxycholic acid ${ }^{+}$or direct stimulation of hepatocyte bicarbonate transport. ${ }^{1+8}$ Unconjugated ursodeoxycholic acid might be excreted by the hepatocyte when supraphysiological doses have been given so that the glycine/taurine conjugation mechanisms are saturated. The fact that no increase in unconjugated ursodeoxycholic acid is found in duodenal bile of patients treated with this compound ${ }^{+9}$ does not contradict this hypothesis, since cholehepatic recycling does not require the unconjugated bile acid to appear in the final bile. ${ }^{+8}$

What relevance does this have to human liver disease? In primary biliary cirrhosis serum concentrations of hydrophobic bile acids, including toxic atypical bile acids, are increased..$^{50-53}$ Administration of ursodeoxycholic acid results in enrichment of the bile acid pool at the expense of endogenous hydrophobic bile acids. The mechanism probably involves competitive inhibition of active terminal ileal transport of conjugates of endogenous bile acids by ursodeoxycholic acid conjugates, thus enhancing faecal excretion of the former. ${ }^{4}$ It seems likely that this is an important component of the mechanism of action of ursodeoxycholic acid in human liver disease, since cirrhotic patients with primary biliary cirrhosis, who, as observed previously, respond poorly to ursodeoxycholic acid treatment, do not show the expected decrease in endogenous bile acids. ${ }^{53}$ The additional protective effects seen in animal and in vitro experiments contribute to the efficacy of ursodeoxycholic acid. In this respect there is emerging evidence that treatment results in more rapid hepatic transit and biliary excretion of endogenous bile acids in primary biliary cirrhosis, as assessed by hepatic handling of the taurocholate analogue selenium homocholic acid taurine $\left({ }^{75} \mathrm{SeHCAT}\right) .{ }^{54}$ The proposed mechanisms of action of ursodeoxycholic acid are summarised in the Figure.

With regard to the hypercholeresis induced by ursodeoxycholic acid, it has been suggested that this could prevent sludging of bile in fine biliary radicles. ${ }^{4}$ This mechanism might be particularly important in patients with cholestatic liver disease associated with cystic fibrosis in whom bile ducts are plugged by viscid mucus. Interestingly, it seems that much higher doses are necessary for an effect in this condition than in other cholestatic diseases. ${ }^{275}$ Some other effects of ursodeoxycholic acid may be relevant clinically. Thus it is known that hepatobiliary transport of organic anions such as bilirubin is enhanced by most bile acids, ' which may explain why many clinical trials have found a reduction in serum bilirubin in patients with primary biliary cirrhosis when on ursodeoxycholic acid treatment (Table). Too heavy a load of 
ursodeoxycholic acid in severely jaundiced patients may have the opposite effect, possibly by non-competitive inhibition of hepatic uptake.' This may in part explain the reduced efficacy of the compound in late stage primary biliary cirrhosis.

Finally, some intriguing work has suggested that ursodeoxycholic acid might have another completely different mechanism of action. It is known that in cholestasis there is an increased expression of HLA class I antigens on hepatocytes and class II antigens on biliary epithelium. Class II antigens are targets for cytotoxic $T$ cell damage. ${ }^{56}$ Class I antigen expression probably also plays a part in cell mediated cytotoxicity. Calmus and his coworkers have found that aberrant class I expression on hepatocytes is reduced by ursodeoxycholic acid treatment, and they speculate that this might lead to a reduction in piecemeal necrosis. ${ }^{57}$ Thus there may be an additional benefit from ursodeoxycholic acid in reducing immunological injury in primary biliary cirrhosis.

\section{Conclusions and speculations}

CURRENT CLINICAL ROLE OF URSODEOXYCHOLIC ACID IN LIVER DISEASE

Ursodeoxycholic acid should now be regarded as having a central role in the medical management of cholestatic liver diseases, in particular primary biliary cirrhosis, primary sclerosing cholangitis, neonatal biliary atresia, and liver disease associated with cystic fibrosis. The reasons for this are the strong evidence that ursodeoxycholic acid improves cholestasis and reduces hepatocellular damage in these conditions; the lack of adverse effects of the compound; and the absence of alternative treatments combining efficacy with lack of adverse effects. The longterm effects on liver histology and prognosis are not yet known, but the available evidence suggests that histology may improve with ursodeoxycholic acid. Caution should be exercised in patients in late stage primary biliary cirrhosis (stages III and IV). The dosage of ursodeoxycholic acid should be around $10 \mathrm{mg} / \mathrm{kg} /$ day for primary biliary cirrhosis and sclerosing cholangitis, ${ }^{58}$ though higher doses may be appropriate in cystic fibrosis. ${ }^{275}$ In chronic active hepatitis available evidence does not presently support a clinical role for ursodeoxycholic acid, though it remains to be determined whether a subsidiary role exists in conjunction with established treatments.

\section{FUTURE RESEARCH}

The results of large multicentre trials of ursodeoxycholic acid currently in progress will be needed before it is known whether liver histology is improved in patients with primary biliary cirrhosis. Longer term controlled studies will then be necessary to determine if the short term effect is maintained and whether there is an effect on prognosis. These could be mounted once the current trials are completed (assuming a beneficial effect in the medium term is confirmed). It seems reasonable to surmise that a beneficial effect on prognosis would be to pro- long the asymptomatic phase of the disease rather than arrest it, slowing progress towards cirrhosis and liver failure. Indeed, recent work shows that, contrary to previously held beliefs, asymptomatic primary biliary cirrhosis is associated with a reduction in life expectancy..$^{59}$ Because of this, and being mindful of the long experience of the use of ursodeoxycholic acid in gall stone disease suggesting a lack of adverse effects, early treatment might be advocated to achieve the maximum impact. This would also be the period in which any effect on reduction of immunological injury would be most productive. Therefore it should not be unethical to mount longer term studies in asymptomatic patients. Given the different mechanisms which are thought to produce liver injury in primary biliary cirrhosis, it may also be appropriate to consider combining a drug specifically targeted at the immune system with ursodeoxycholic acid in future trials.

Other cholestatic liver diseases, in particular those associated with primary sclerosing cholangitis and cystic fibrosis, are at present virtually untreatable, so large multicentre controlled trials are urgently needed. Since these diseases share a common feature with primary biliary cirrhosis, namely accumulation of toxic hydrophobic bile acids, it is not unreasonable to hope that ursodeoxycholic acid treatment will be beneficial. It will be interesting to discover whether hypercholeresis has any relevance to clinical efficacy, particularly in cystic fibrosis. It would be useful to know whether ursodeoxycholic acid is of value in chronic hepatitis when given in combination with interferon alfa.

The emergence of ursodeoxycholic acid as a promising new treatment in a variety of chronic liver diseases is not only a hopeful development for clinical practice but also provides a stimulus to further research into the underlying mechanisms of liver damage in cholestasis. The next few years should provide some exciting new insights, together with detailed clarification of the clinical role, applications, and mechanisms of action of the compound.

1 Kitani K. Hepatoprotective effect of ursodeoxycholic acid in experimental animals. In: Paumgartner G, Stiehl A, Barbar L, Roda E, eds. Strategies for the treatment of hepatobiliary diseases. Dordrecht: Kluwer Academic, 1990: 43-56.

2 Hofmann AF. Bile acid hepatoxicity and the rationale of UDCA therapy in chronic cholestatic liver disease: some hypotheses. In: Paumgartner G, Stiehl A, Barbara L, Rod $\mathrm{E}$, eds. Strategies for the treatment of hepatobiliary diseases. Dordrecht: Kluwer Academic, 1990: 13-33.

3 Leuschner U, Leuschner M, Sieratzki J, Kurtz W, Hubner K. Gallstone dissolution with ursodeoxycholic acid in patients with chronic active hepatitis and two year follow up: a pilot with chronic active hepatitis and tw
study. Dig Dis $S c i$ 1985; 30: 642-9.

4 Hofmann AF, Popper H. Ursodexoycholic acid for primary biliary cirrhosis. Lancet 1987; ii: 398-9.

5 Triger DR. Update on primary biliary cirrhosis. Dig Dis 1990 8: 61-70.

6 Kaplan MM. Medical treatment of primary biliary cirrhosis Semin Liver Dis 1989; 9: 138-43.

7 Graham JM, Northfield TC. Solubilisation of lipids from hamster bile canalicular and contiguous membranes and from human erythrocyte membranes by conjugated bile salts. Biochem $\mathcal{F}$ 1987; 242: 825-34.

8 Fisher MM, Paradine ME. Influence of ursodeoxycholic acid on biochemical parameters in cholestatic liver disease [Abstract]. Gastroenterology 1986; 90: 1725 .

9 Poupon R, Chrétien Y, Poupon RE, et al. Is ursodeoxycholic acid an effective treatment for primary biliary cirrhosis?
Lancet 1987; ii: $834-6$.

10 Lotterer E, Bauer FE, Bircher J. Safety of ursodeoxycholic acid in chronic cholestasis [A bstract]. Hepatology 1988; 8: acid in

11 Hayashi $\mathrm{H}$, Higuchi $\mathrm{T}$, Ichimaya $\mathrm{H}$, et al. Ursodeoxycholic acid reduces secondary liver damage in primary biliary 
cirrhosis and primary sclerosing cholangitis [Abstract]. Hepatology 1988; 8: 1417.

12 Bateson MC, Ross PE, Diffey BL. Ursodeoxycholic acid in primary biliary cirrhosis. Lancet 1989; i: 898-9.

13 Matsuzaki Y, Tanaka N, Osuga T, et al. Improvement of biliary enzyme levels and itching as a result of long term administration of ursodeoxycholic acid in primary biliary cirrhosis. Am $\mathcal{F}$ Gastroenterol 1990; 85: 15-23.

14 Osuga T, Tanaka N, Matsuzaki Y, Aikawa T. Effect of ursodeoxycholic acid in chronic hepatitis and primary biliary cirrhosis. Dig Dis Sci 1989; 34 (suppl): 49-51.

15 Leuschner U, Fischer H, Kurtz W, et al. Ursodeoxycholic acid in primary biliary cirrhosis: results of a double blind in primary biliary cirrhosis: results of a doub

16 Hadziyannis SJ, Hadziyannis ES, Makris A. A randomized controlled study of ursodeoxycholic acid in primary biliary controlled study of ursodeoxycholic acid in primas
cirrhosis [Abstract]. Hepatology 1989; 10: 580 .

17 Podda M, Battezzati PM, Crosignani A, et al Ursodeoxycholic acid for symptomatic primary biliary cirrhosis: a double blind multicenter study [Abstract]. Hepatology 1989; 10: 639 .

18 O'Brien CB, Senior JR, Sternlieb JM, et al. Ursodiol in the treatment of primary biliary cirrhosis. Gastroenterology 1990 98: A617.

19 Roseau G. Acide ursodéoxycholique dans la cirrhose bilaire primitive: première étude en double aveugle [Abstract] Presse Med 1990; 16: 237.

20 Toda G, Oka H, Hasumura $\mathrm{Y}$, et al. A multicenter double blind controlled trial of ursodeoxycholic acid for primary biliary cirrhosis in Japan. In: Meeting Handbook, XIth biliary cirrhosis in Japan. In: Meeting Handbook,
International Bile Acid Meeting (Poster Abstract No 76) Freiburg, Germany: Falk symposium No 58, 1990.

21 Poupon RE, Eschwege E, Poupon R, UDCA-PBC study group. Ursodeoxycholic acid for the treatment of primary biliary cirrhosis: interim analysis of a double blind multicentre randomised trial. F Hepatol 1990; 11: 16-21.

22 Vogel $W$, Kathrein $H$, Judmaier $G$, Braunsteiner $H$ Deterioration of primary biliary cirrhosis during treatmen with ursodeoxycholic acid. Lancet 1988; i: 1163.

23 Raedsch R, Stiehl A, Theilmann L, et al. Influence of urosdeoxycholic acid on primary biliary cirrhosis depending urosdeoxycholic a aid on primary biliory cirrhosis: Aepen.
on stage of the disease. Gastroenterology 1989; 96 : A647.

24 O'Brien CB, Senior JR, Batta AK, et al. Ursodeoxycholic acid treatment produces marked clinical and biochemical amelioration of primary sclerosing cholangitis. Gastroamelioration of primary

25 Chazouillières O, Poupon R, Capron JP, et al. Ursodeoxycholic acid for primary sclerosing cholangitis. $\mathcal{F}$ Hepatol 1990; 11: 120-3.

26 Stiehl A, Raedsch R, Rudolph G, Thielmann L. Treatment of primary sclerosing cholangitis with ursodeoxycholic acid: first results of a controlled study [Abstract]. Hepatology 1989; 10: 602 .

27 Cotting J, Lentze MJ, Reichen J. Effects of ursodeoxycholic acid treatment on nutrition and liver function in patients with cystic fibrosis and longstanding cholestasis. Gut 1990; with cystic $918-21$.

28 Bittner P, Sailer T, Ott H, et al. The effect of treatment with ursodeoxycholic acid in cystic fibrosis with hepatopathy:
results of a placebo controlled study. In: Meeting Handbook, XIth Intermational Bile Acid Meeting [Abstract]. Freiburg, Germany: Falk symposium No 58, 1990: 67.

29 Thompson JN, Cohen J, Blenkham JI, et al. A randomised clinical trial of ursodeoxycholic acid in odstructive jaundice. BrF Surg 1986; 73: 634-6.

30 Levy J, De Felice A, Ramakrishnan R, et al. Short term effects of ursodeoxycholic acid in chronic cholestatic disorders of childhood. Gastroenterology 1990; 98: A603.

31 Ullrich D, Rating D, Schroter W, Hanefeld F, Bircher J. Treatment with ursodeoxycholic acid renders children with bilary atresia suitable for liver transplantation. Lancet 1987; ii: 1324 .

32 Nittono H, Tokita A, Hayashi M, et al. Ursodeoxycholic acid in biliary atresia. Lancet 1988; i: 528 .

33 Nittono H, Tokito A, Hayashi M, et al. Ursodeoxycholic acid therapy in the treatment of biliary atresia. Biomed Pharmacother 1989; 43: 37-41.

34 A-Kader HH, Heubi JE, Setchell KDR, Ryckman FC, Balistreri WF. The effects of ursodeoxycholic acid therapy in with extrahepatic biliary atresia. Gastroenterology 1990 98: A564

35 Reyes H, Palma J, Ribalta J, et al. Effects of ursodeoxycholic acid in patients with cholestasis of pregnancy. In: Meetin Handbook, XIth International Bile Acid Meeting. Freiburg, Germany: Falk symposium No 58, 1990: 62 .

36 Birchner J. Treatment of patients with benign recurrent intrahepatic cholestasis. Hepatology 1989; 10: 1030 .

37 Bellentani S, Tabarroni G, Barchi T, et al. Effect of ursodeoxycholic acid on alanine aminotransferase and $\because$-glutamyl transferase serum levels in patients with hyper transaminasemia. $\mathcal{J}$ Hepatol $1989 ; 8: 7-12$.

38 Ideo G, Bellati G, Bottelli $R$, Pedraglio E. Treatment of non-A non-B chronic hepatitis with ursodeoxycholic acid: results from a prospective double blind controlled trial. In: Meeting Handbook, XIth International Bile Acid Meeting (Poster Abstract No 72). Freiburg, Germany: Falk symposium No 58,1990 .

39 Quist R, Hofmann AF, Barrett KE. Activation of mast cells by bile acids. Gastroenterology 1989; 96: A691.

40 Salvioli G, Lugli R, Pradelli JM. Effects of bile salts on membranes. In: Calandra S, Carulli N, Salvioli G, eds. Liver and lipid metabolism. Amsterdam: Excerpta Medica, 1984: 163-79.

41 Heuman DM, Komito SF, Pandak WM, Hylemon PB, Vlahcevic ZR. Tauroursodeoxycholate protects against cholestatic and hepatocytolytic toxicity of more hydrophobic bile salts. Gastroenterology 1989; 96: A607.

42 Galle PR, Theilmann L, Kohl B, Raedsch R, Stiehl A. Ursodeoxycholate reduces toxicity of different bile acids in human primary hepatocyte cultures. Gastroenterology 1989; 96: A599.

43 Schmucker DL, Kitani K, Ohta M, Kanai S, Sato Y. Hepatic injury induced by bile salts and its prevention by tauroursodeoxycholate: correlation between biochemical and morphological events [Abstract]. Hepatology 1988; 8: 1357.

44 Kitani K, Kanai S. Tauroursodeoxycholate prevents taurocholate induced cholestasis. Life Sci 1982; 30: 515-23.

45 Kitani K, Kanai S. Interactions between different bile salts in the biliary excretion. Res Commun Chem Pathol Pharmacol 1983; 39: 139-52.

46 Kitani K, Ohta M, Kanai S. Tauroursodeoxycholate prevents biliary protein excretion induced by other bile salts in the rat. Am F Physiol 1985; 248: 407-17.

47 Heuman DM, Conroy RM. Tauroursodeoxycholate protects cholesterol rich model membranes against disruption by more hydrophobic bile salts. In: Meeting Handbook, XIth International Bile Acid Meeting. Freeiburg, Germany: Falk International Bile Acid Meeting.

48 Erlinger S, Dumont M. Influence of ursodeoxycholic acid on bile secretion. In: Paumgartner G, Stiehl A, Barbara L, Roda E, eds. Strategies for the treatment of hepatobiliary diseases. Dordrecht: Kluwer Academic, 1990: 35-42.

49 Crosignani A, Battezzati PM, Camisasca M, et al. Changes in bile acid composition induced in patients with primary biliary cirrhosis by ursodeoxycholic acid administration. In: Meeting Handbook, XIth International Bile Acid Meeting (Poster Abstract No 67). Freiburg, Germany: Falk symposium No 58, 1990.

50 Chrétien Y, Poupon R, Gherardt MF, et al. Bile acid glycine and taurine conjugates in serum of patients with primary biliary cirrhosis. Gut 1989; 30: 1110-5.

51 Roda E, Mazzella G, Bazzoli F, et al. Effect of ursodeoxycholic acid administration on biliary lipid secretion in primary biliary cirrhosis. Dig Dis Sci 1989; 34 (suppl): 52-8.

52 Batta AK, Salen G, Arora R, et al. Effect of ursodeoxycholic acid on bile acid metabolism in primary biliary cirrhosis. Hepatology 1989; 10: 414-9.

53 Stiehl A, Rudolph G, Raedsch R, et al. Ursodeoxycholic acidinduced changes of plasma and urinary bile acids in patients with primary biliary cirrhosis. Hepatology 1990; 12: 492-7.

54 Jazrawi RP, de Caestecker JS, Brittain A, et al. Effect of ursodeoxycholic acid on hepatic bile acid handling in ursodeoxycholic acid on hepatic bile acid
primary biliary cirrhosis. Gut 1990; 31: A1184.

55 Colombo C, Setchell KDR, Podda M, et al. Ursodeoxycholic acid in patients with cystic fibrosis and liver disease: a doseresponse study. In: Meeting Handbook, XIth International Bile Acid Meeting (Poster Abstract No 66). Freiburg, Germany: Falk symposium No 58, 1990

56 Pape GR, Spengler U, Jung MC, et al. Natural history and pathogenesis of primary biliary cirrhosis. In: Paumgartner G, Stiehl A, Barbara L, Roda E, eds. Strategies for the treatment of hepatobiliary diseases. Dordrecht: Kluwer treatment of hepatobilize.

57 Calmus Y, Gane P, Rouger P, Poupon R. Hepatic expression of class I and II major histocompatibility complex molecules in primary biliary cirrhosis: effect of ursodeoxycholic acid Hepatology 1990; 11 : 12-5.

58 Podda M, Ghezzi C, Battezzati PM, et al. Effect of different doses of ursodeoxycholic acid in chronic liver disease. Dig Dis Sci 1989; 34: 59-65S

59 Balasubramanian K, Grambsch PM, Wesner RH, Lindor KD, Dickson ER. Diminished survival in asymptomatic primary biliary cirrhosis: a prospective study. Gastroenterology 1990; 98: 1567. 